\title{
Signal processing algorithm of ship navigation radar based on azimuth distance monitoring
}

\author{
Yuxin Qin ${ }^{*}$ and Yu Chen \\ Zhengzhou University of Aeronautics, Zhengzhou, Henan 450046, PR China
}

Received: 20 July 2019 / Accepted: 30 August 2019

\begin{abstract}
The effect of ship navigation radar signal processing has a great impact on the overall performance of the radar system. In this paper, the signal processing algorithm is studied. Firstly, the principle of radar azimuth and distance monitoring is introduced, then the pulse accumulation algorithm and median filtering algorithm are analyzed, and finally a sea clutter suppression algorithm based on sensitivity time control (STC) and a rain and snow clutter suppression algorithm based on constant false alarm rate are designed to improve the target monitoring performance of radar. In the test of the algorithm, the radar signal processing algorithm designed in this study has good precision as monitoring error of the target's azimuth and distance is controlled within 1\%; and it also has a better suppression effect of sea clutter and rain and snow clutter, which can suppress the clutter well, improve the target clarity, and ensure the safe navigation of the ship. The experiment proves the effectiveness of the proposed algorithm and provides some theoretical basis for the better processing of radar signals, which is beneficial to improve the environment perception ability of ships in harsh environments and promote the further development of the navigation industry.
\end{abstract}

Keywords: Ship navigation radar signal processing / clutter suppression / median filter / monitoring performance

\section{Introduction}

Radar achieves the positioning and detection of an object by directionally launching electromagnetic energy, receiving the reflected electric wave of the object, and calculating the direction, speed and shape of the object, which has been widely used in fields, such as military [1], remote sensing [2], aviation [3] and navigation. With the development of shipping, ship radar has gradually gained popularity [4]. The ship navigation radar is installed on the ship for positioning, navigation, obstacle avoidance, etc. It is the most advantageous tool for azimuth and distance monitoring when the ship is sailing, which can effectively ensure the safety of the ship. The processing of radar signal is the key to radar performance and has important research value. With the development of technology, radar signal processing has evolved from traditional analog technology to digital signal processing (DSP). Xin et al. [5] studied the problem of weak radar angular resolution and transformed it into a constrained optimization problem. A model based on singular value decomposition was designed, and the optimal solution was found by the interior point method.

* Corresponding author: yuxqinyx@yeah.net
The simulation experiment suggested that the model had good resolution performance and improved the resolution of the radar signal by 4 times. Yi et al. [6] designed an improved orthogonal weighting (OW) algorithm to monitor the targets in sea clutter and proved the effectiveness of the proposed method through simulation experiments. $\mathrm{Wu}$ et al. [7] proposed an algorithm based on Hilbert transform theory. Firstly, the radar signal image was decomposed into a single-wave periodic image and then calculated by Hilbert transform. Cai et al. [8] analyzed the weak target detection of the sea surface, designed a time-frequency distribution (TFD) fusion strategy method based on the population evolution algorithm, and extracted the normalized frequency edge features to reduce the feature dimension. The experimental results showed that the method could improve the radar's weak target detection ability in any environment. At present, most of the radar used in ship navigation is analog radar, which is inconvenient to debug and has poor anti-jamming ability. Moreover, the development of radar digital processing is still slow. The main research directions include digital circuit hardware, digital signal processing and digital fusion. Some achievements have also been made, but generally speaking, the overall technical level of radar signal processing is still low. In this paper, the signal 
processing algorithms of radar are studied. The pulse accumulation, and median filtering and sea clutter, rain and snow clutter suppression algorithms are emphatically introduced, and the effectiveness of these algorithms is proved by experiments. Compared to other algorithms, they have smaller monitoring errors and better inhibition effects. Due to these algorithms, the radar image quality is significantly improved, which is conducive to the safe navigation of ships, the development of radar market in China, and further application of digital radar in the navigation field. This work makes some contributions to the further development of ship navigation radar.

\section{Ship navigation radar}

The pulse radar is generally used on the ship, which can transmit pulse modulation signals to form pulses and improve radar monitoring accuracy. The working principle of the radar is: the pulse modulator provides the modulation pulse for the transmitter, and the transmitter acts as the stable frequency source of the radar, which can ensure the coherent signal required by the radar. When the pulse is transmitted, the transceiver switch will cut off the receiving branch and then disconnect the transmitting branch at the end of the pulse, and the echo signal received by the antenna is connected to the receiving branch. The receiver mixes the received carrier frequency and the local oscillator signal to obtain an intermediate frequency signal and performs digital processing to obtain useful information displayed in the display. The timer provides the beat for the radar extension through the departure pulse to ensure the normal operation of the radar.

The radar tracking and alarming of the target is mainly realized by two functions, that is, the azimuth monitoring and distance monitoring of the target. Azimuth monitoring can be achieved by a sharp azimuth beam of the radar antenna, and distance monitoring is achieved by calculating the time of the pulse round trip. The details are shown below.

\subsection{Principle of radar azimuth monitoring}

The radar's azimuth monitoring relies on the ability of the electromagnetic energy to gather in the direction, that is, the beam width. The beam is divided into the main lobe and the side lobe. The energy is mainly concentrated on the main lobe. When the main lobe beam is aimed at the target, the strongest echo signal can be received, and the direction of the beam axis is the target direction, as shown in Figure 1.

\subsection{Principle of radar distance monitoring}

The theoretical basis of radar distance monitoring is the nature of electromagnetic wave propagating in a uniform medium at a uniform speed. When an object is encountered, the electromagnetic wave will reflect the radar signal. It is assumed that the round trip time of the electromagnetic wave is $\Delta t$, and the propagation speed of electromagnetic wave in the air is $v\left(\operatorname{approx} .3 \times 10^{8} \mathrm{~m} / \mathrm{s}\right)$.

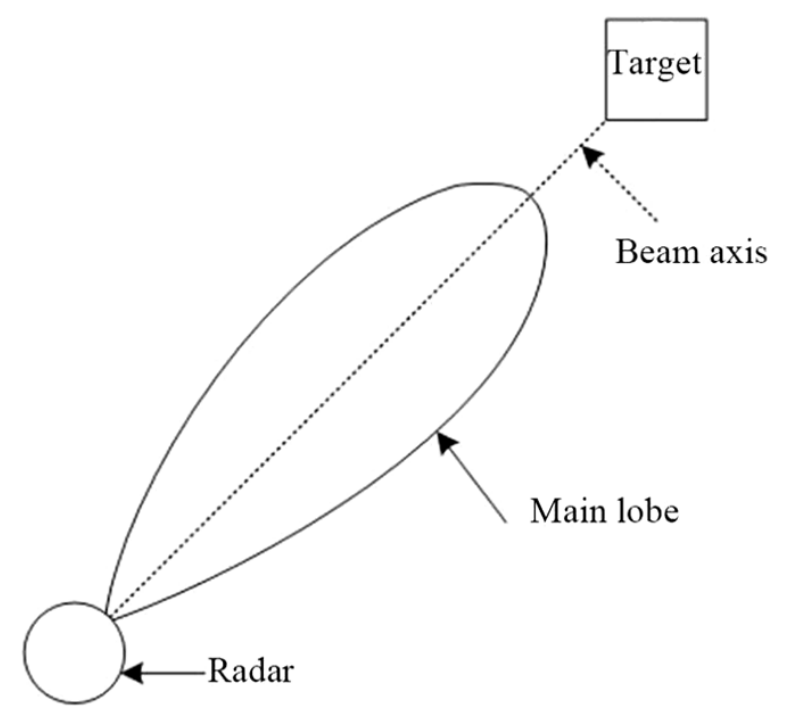

Fig. 1. Radar azimuth monitoring.

The calculation formula of distance between the target and radar is:

$$
R=v \frac{\Delta t}{2}
$$

\section{Radar signal processing algorithm}

When there is interference clutter in the working range of the radar, the radar can separate the detected target according to the difference of Doppler frequency. However, when the interference clutter is too strong, the performance of the radar will be affected. In order to achieve accurate monitoring of the target, an efficient signal processing method is required.

\subsection{Pulse accumulation algorithm}

In the course of working, the radar usually launches multiple radars to perform multiple measurements to improve the signal-to-noise ratio of the echo signal and enhance the detection capability of the target [9]. The formula for calculating the number of echoes is:

$$
n=\frac{\theta T f_{r}}{2 \pi}
$$

where $\theta$ represents the width angle of the radar lobe, $T$ indicates the scan time, and $f_{r}$ indicates the pulse repetition frequency. The superposition of multiple pulse echoes is pulse accumulation, which can be calculated by superposition averaging:

$$
y(t)=\frac{\sum_{i=1}^{N}\left(s_{i}(t)+n_{i}(t)\right)}{N},
$$


where $s(t)$ represents an echo signal, $n(t)$ indicates clutter noise, and $i$ indicates the number of echoes, $i=1,2, \ldots, N$.

\subsection{Median filtering algorithm}

The median filtering algorithm is used to filter out noise and protect the edge of the signal [10], which can effectively improve the accuracy of radar target monitoring. In a filter window with a length of $L=2 N+1$, it is assumed that the radar signal of the positive electricity in the window is $x(n-N), \ldots, x(n), \ldots, x(n+N)$ at moment $n$, and the output of the median filter [11] can be expressed as:

$$
y(n)=\operatorname{med}[x(n-N), \ldots, x(n), \ldots, x(n+N)],
$$

where med \lfloor\rfloor indicates the result of removing the median value after the number in the window is incremented. When the window is $2 N+1$, a pulse with a width less than or equal to $N$ will be cleared to achieve noise removal.

\subsection{Sea clutter suppression algorithm}

When the radar pulse is reflected at a close sea level, it will cause sea clutter, which is one of the interference sources of the radar signal [12]. Sea clutter will affect the monitoring performance of the radar, but can be suppressed by the STC circuit [13].

The radar signal is expressed by $S_{l}$, and then the output of the STC can be expressed as:

$$
S_{s t c}=S_{l} \cdot F(R)
$$

where $F(R)$ indicates the gain of STC, $F(R)=R^{\alpha}$, and $\alpha$ indicates the adjustment factor.

The radar receiver processes the signal, and the processed signal is:

$$
l g\left(S_{s t c}\right)=\lg \left(S_{l}\right)+\alpha \lg R .
$$

\subsection{Rain and snow clutter suppression algorithm}

In the bad weather conditions such as rain and snow, the performance of the radar will be greatly affected [14], which is because that rain and snow clutter will appear when the radar pulse encounters rain, snow, hail, etc. The greater the rain and snow is, the stronger the clutter is, but it can be suppressed by the constant false alarm rate (CFAR) [15]. Generally, rain and snow clutter is evenly distributed. In low-resolution radars, the probability density of clutter conforms to the Rayleigh distribution, and the total echo voltage can be expressed as the sum of two orthogonal terms:

$$
u_{\text {total }}^{2}=u_{x}^{2}+u_{y}^{2}
$$

The probability density distribution of voltage $u_{x}$ and $u_{y}$ can be expressed as:

$$
\rho\left(u_{x}\right)=\frac{1}{\sqrt{2 \pi} \varepsilon_{x}} \exp \left[-\frac{u_{x}^{2}}{2 \varepsilon_{x}^{2}}\right],
$$

$$
\rho\left(u_{y}\right)=\frac{1}{\sqrt{2 \pi} \varepsilon_{y}} \exp \left[-\frac{u_{y}^{2}}{2 \varepsilon_{y}^{2}}\right],
$$

where $\varepsilon_{x}$ and $\varepsilon_{y}$ respectively represent the variances of $u_{x}$ and $u_{y}$.

The joint probability density of them can be expressed as:

$$
\rho\left(u_{x}, u_{y}\right)=\frac{1}{2 \pi \varepsilon^{2}} \exp \left[-\frac{u_{x}^{2}+u_{y}^{2}}{2 \varepsilon^{2}}\right] .
$$

The probability density of clutter can be expressed as:

$$
\rho\left(u_{\text {total }}\right)=\frac{u_{\text {total }}}{\varepsilon^{2}} \exp \left[-\frac{u_{\text {total }}^{2}}{2 \varepsilon^{2}}\right] .
$$

In high-resolution radars, the probability density can be expressed by the Weibull distribution [16]:

$$
\rho\left(u_{\text {total }}\right)=\left(\frac{\eta}{\sigma}\right)\left(\frac{u_{m}}{\sigma}\right)^{\eta-1} \exp \left[-\left(\frac{u_{\text {total }}}{\sigma}\right)^{\eta}\right]
$$

where $\eta$ represents the shape scale parameter and $\sigma$ indicates the intensity scale parameter.

CFAR can adjust the monitoring threshold by clutter, thus ensuring a certain false alarm rate and improving the monitoring ability of the target. In the processing of constant false alarm rate, if there is a large noise fluctuation, the false alarm probability will increase. In order to maintain the constant false alarm rate, it is necessary to appropriately increase the detection threshold and improve the input signal-to-noise ratio, i.e., constant false alarm rate loss $L_{\text {cfar. }}$

In rain and snow clutter, the threshold is set as $C_{T}$, and the probability that the clutter amplitude exceeds the threshold can be expressed as:

$$
\rho=\exp \left(\frac{C_{T}^{2}}{2 \varepsilon^{2}}\right) .
$$

In the constant false alarm monitor, CA-CFAR [17] is selected to suppress rain and snow clutter, and the calculation formula is:

$$
Z=\frac{1}{2 n} \sum_{i=1}^{2 n} X(i)
$$

\section{Algorithm test}

The FURUNO radar antenna (Fig. 2) is used to carry out the simulation experiment to test the signal processing algorithm. The frequency of the radar antenna is $9410 \pm$ $30 \mathrm{MHz}$. The horizontal and vertical beam widths are 2.4 degrees and 27 degrees respectively. The rotational speed is $25 \mathrm{rad} / \mathrm{min}$. The testing spot was the dynamic and static testing spot of a port. The azimuth distance monitoring and sea clutter suppression functions of the radar were verified. 


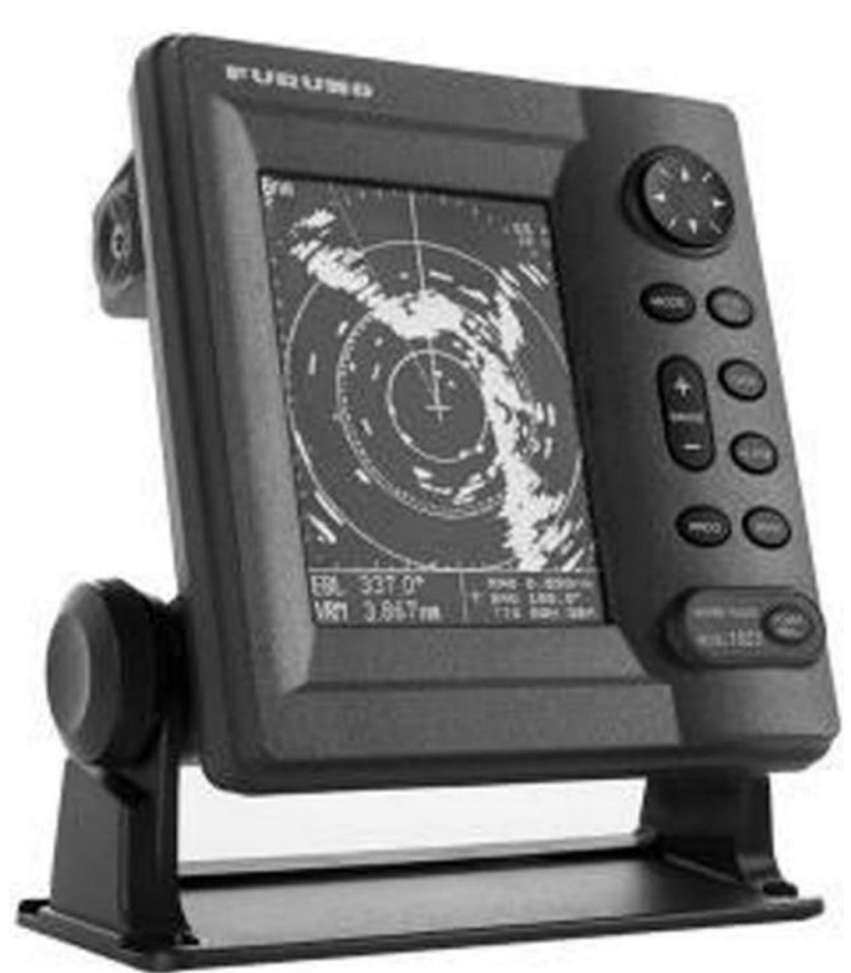

Fig. 2. Radar antenna of FURUNO.

\subsection{Azimuth distance monitoring test}

Ten obstacles are arranged on the sea surface as radar monitoring objects to test the radar's azimuth and distance monitoring performance. The actual azimuth and distance between the obstacle and radar are shown in Table 1 .

After the pulse accumulation and median filtering algorithm is processed, the obtained radar monitoring results are shown in Table 2.

According to Table 2, the radar has shown good performance in the monitoring of obstacles with different orientations and different distances. The minimum error of azimuth monitoring is $0.18 \%$, the maximum is $0.85 \%$, and both are within $1 \%$. The minimum error of distance monitoring is $0.19 \%$ and the maximum is $0.84 \%$, both of which are controlled within $1 \%$, indicating that it has good accuracy in azimuth distance monitoring.

\subsection{Sea clutter suppression test}

Sea clutter is the main clutter in the radar signal, and its suppression can effectively improve the signal quality. When the clutter suppression intensity is 0 , the radar echo signal is shown in Figure 3.

In Figure 3, the signal is subject to large interference so that the orientation and distance of the target cannot be effectively recognized. When the suppression intensity is manually adjusted to 60 , the echo signal is shown in Figure 4.
Table 1. Actual orientation and distance of the monitored object.

\begin{tabular}{lll}
\hline Obstacle number & Orientation & Distance \\
\hline 1 & $27.3^{\circ}$ & $136.8 \mathrm{~m}$ \\
2 & $56.2^{\circ}$ & $129.7 \mathrm{~m}$ \\
3 & $15.6^{\circ}$ & $159.2 \mathrm{~m}$ \\
4 & $23.4^{\circ}$ & $59.7 \mathrm{~m}$ \\
5 & $11.7^{\circ}$ & $62.1 \mathrm{~m}$ \\
6 & $49.6^{\circ}$ & $36.8 \mathrm{~m}$ \\
7 & $52.3^{\circ}$ & $168.2 \mathrm{~m}$ \\
8 & $19.8^{\circ}$ & $119.7 \mathrm{~m}$ \\
9 & $26.7^{\circ}$ & $94.6 \mathrm{~m}$ \\
10 & $51.4^{\circ}$ & $79.5 \mathrm{~m}$ \\
\hline
\end{tabular}

Table 2. Radar monitoring results.

\begin{tabular}{lllll}
\hline Obstacle number & Orientation & Error & Distance & Error \\
\hline 1 & $27.1^{\circ}$ & $0.73 \%$ & $136.5 \mathrm{~m}$ & $0.22 \%$ \\
2 & $56.3^{\circ}$ & $0.18 \%$ & $129.3 \mathrm{~m}$ & $0.31 \%$ \\
3 & $15.5^{\circ}$ & $0.64 \%$ & $158.9 \mathrm{~m}$ & $0.19 \%$ \\
4 & $23.2^{\circ}$ & $0.85 \%$ & $59.2 \mathrm{~m}$ & $0.84 \%$ \\
5 & $11.6^{\circ}$ & $0.85 \%$ & $62.3 \mathrm{~m}$ & $0.32 \%$ \\
6 & $49.4^{\circ}$ & $0.40 \%$ & $36.5 \mathrm{~m}$ & $0.82 \%$ \\
7 & $51.9^{\circ}$ & $0.76 \%$ & $167.8 \mathrm{~m}$ & $0.24 \%$ \\
8 & $19.7^{\circ}$ & $0.51 \%$ & $118.9 \mathrm{~m}$ & $0.67 \%$ \\
9 & $26.5^{\circ}$ & $0.75 \%$ & $94.2 \mathrm{~m}$ & $0.42 \%$ \\
10 & $51.2^{\circ}$ & $0.39 \%$ & $79.2 \mathrm{~m}$ & $0.38 \%$ \\
\hline
\end{tabular}

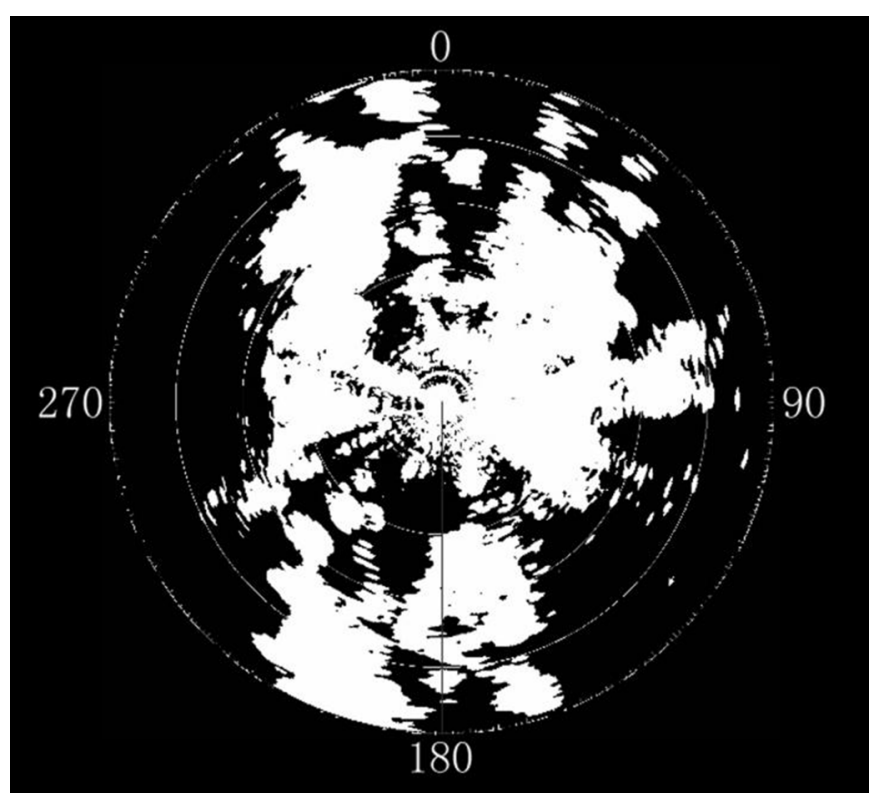

Fig. 3. Echo signal diagram before suppression. 


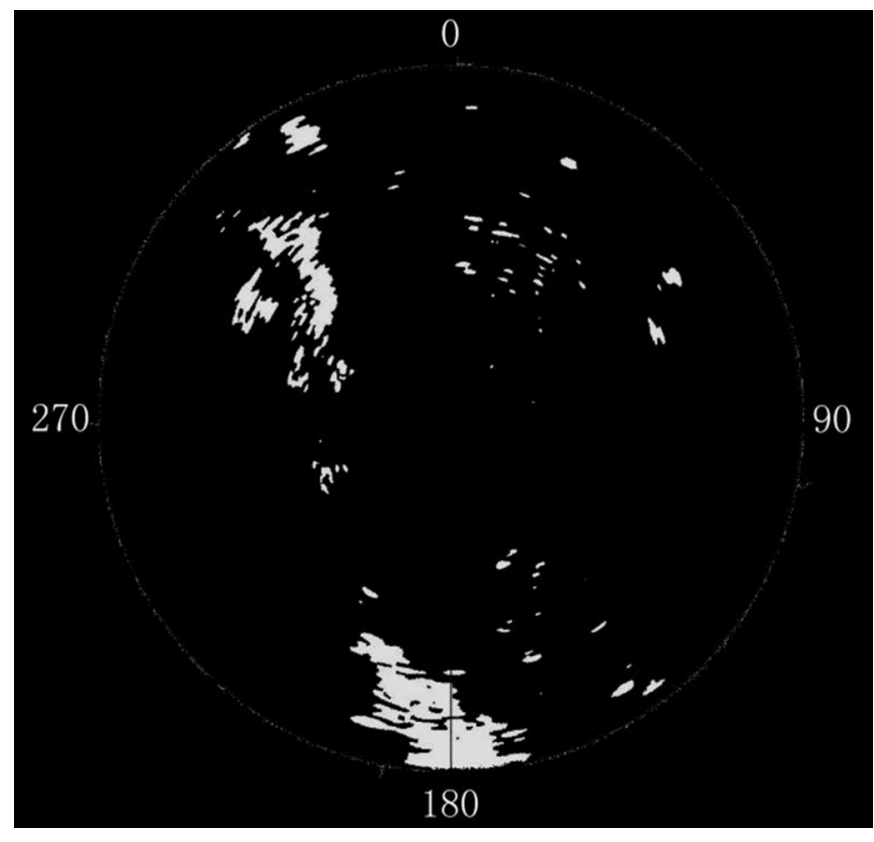

Fig. 4. Sea clutter suppression image.

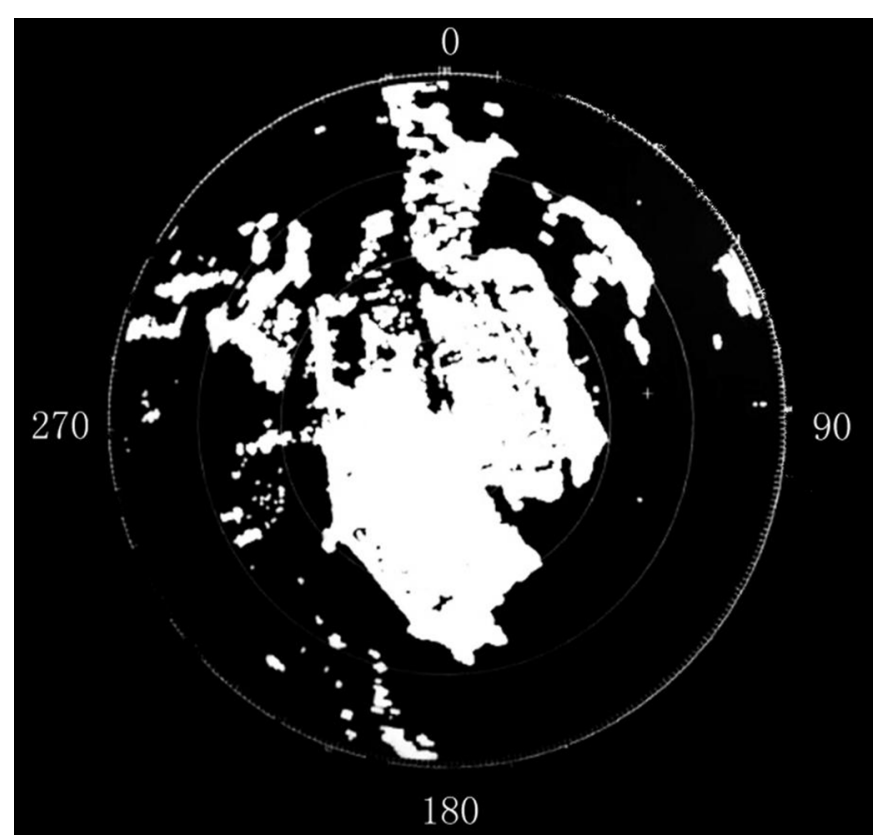

Fig. 5. Echo signal diagram before suppression.

From Figure 4, it can be seen that under the suppression intensity of 60 , the clutter is effectively suppressed and the target is more clearly visible. It shows that the suppression algorithm based on STC in this paper is effective and can improve the accuracy of target recognition.

\subsection{Rain and snow clutter suppression test}

Figure 5 shows the echo image of the radar signal when rain and snow clutter suppression is not turned on. It can be

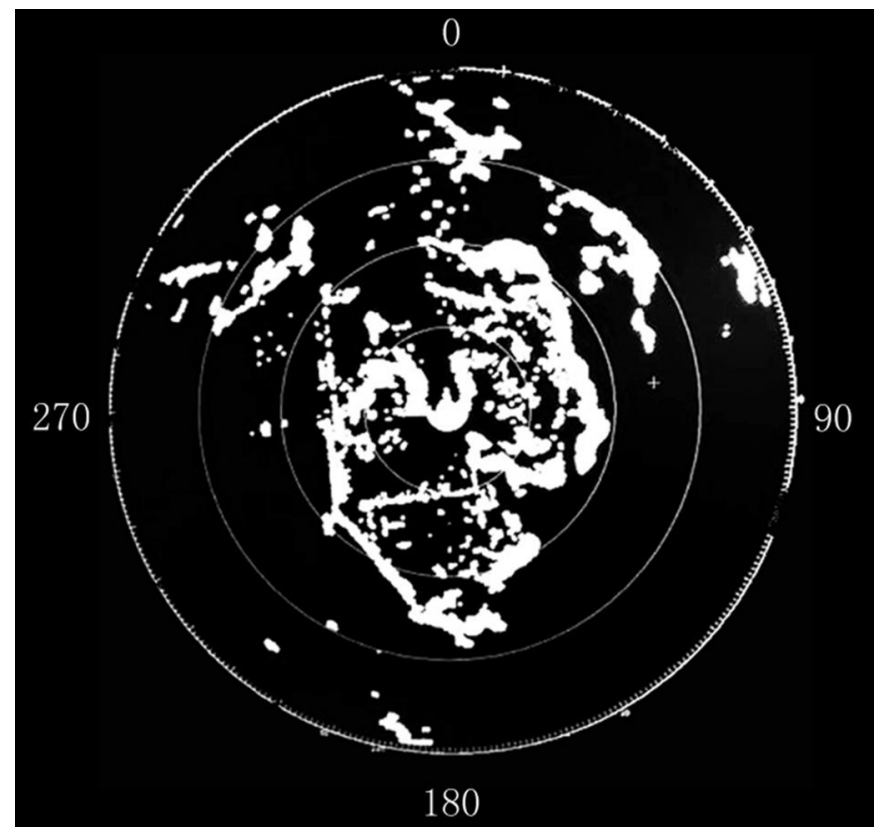

Fig. 6. Rain and snow clutter suppression image.

found that under the action of rain and snow, the target in the echo signal appears in blocks, which cannot be accurately identified.

The rain and snow clutter is suppressed by using the CA-CFAR method proposed in this study, and the results are shown in Figure 6. It can be found that the target block in the original image has been basically eliminated, and the small target has been clearly displayed, which indicates that the rain and snow clutter suppression algorithm in this study is reliable.

\section{Discussion and conclusion}

The ship navigation radar is an indispensable tool for ships offshore operations. By processing the radar signal, it can monitor the azimuth and distance of the target to avoid collision [18], thus ensuring the safe navigation of the ship [19]. The electromagnetic waves generated by the radar can penetrate the clouds, rain and snow and detect the reefs, icebergs, ships, etc. on the sea through the returned signal, playing a role in navigation and obstacle avoidance. In radar signal processing, the problems of how to suppress signal interference, improve the ability to monitor targets, and reduce errors and omissions during monitoring are urgently needed to be solved.

The performance of the propagation navigation radar has been improved due to the development of digital radar. It has high stability and anti-interference and is easy to install, especially in the algorithm processing, which has the advantage that the analog radar does not have. In this paper, firstly, the principle of radar azimuth and distance monitoring is analyzed, and then the radar signal processing algorithm is studied. The pulse accumulation algorithm, median filtering algorithm and sea clutter, rain and snow clutter suppression algorithm are analyzed. 
Both pulse accumulating and median filtering algorithms are designed to reduce noise, improve signal-to-noise ratio, and enhance radar's target monitoring capability; the sea clutter suppression algorithm is used with a method based on STC, and the rain and snow clutter suppression algorithm is used with a method based on constant the false alarm rate. In order to understand the performance of the algorithms, the FURUNO radar is used for testing, and it can be found from the test results that the algorithms proposed in this study are effective. Firstly, in the monitoring of 10 obstacles with different azimuths and distances, the radar monitoring results show a small error, and the azimuth monitoring error and distance monitoring error are both controlled within 1\%, indicating that the signal processing algorithm of this paper can effectively improve the monitoring accuracy of the radar. Then, in the perspective of the suppression of sea clutter and rain and snow clutter, before the suppression, the recognition of the target is seriously disturbed by the existence of clutter; under the action of the suppression algorithm, the clutter is well suppressed, which makes the target clearer and easier to monitor and significantly enhances radar's monitoring performance.

In summary, the radar signal processing algorithm designed in this study has high reliability as it can improve the monitoring performance of the target, reduce the monitoring error, and improve the monitoring ability of the ship under severe weather conditions and the safety of the ship sailing. This study makes some contributions to promote the development of the navigation industry.

\section{References}

1. K.E. Olsen, W. Asen, IEEE Aerosp. Electron. Syst. Mag. 32, 4-12 (2017)
2. P. Broche, P. Forget, J.C.D. Maistre, J.L. Devenon, M. Crochet, Radio Sci. 22, 69-75 (2016)

3. A.F. Scannapieco, A. Renga, G. Fasano, A. Moccia, ISPRS XLII-2/W6, 333-338 (2017)

4. G. Galati, G. Pavan, F. De Palo, G. Ragonesi, J. Navig. 70, 1098-1116 (2017)

5. X. Zhang, X.M. Liu, C. Liu, J. Dalian Maritime Univ. 43, 45-52 (2017)

6. C. Yi, Z. Ji, J. Xie, M. Sun, Y. Li, IET Radar Sonar Navig. 10, 107-113 (2016)

7. L.C. Wu, D.J. Doong, J.H. Wang, IEEE Geosci. Remote Sens. 14, 644-648 (2017)

8. Z.H. Cai, M. Zhang, Y.J. Liu, IET Radar Sonar Navig. 12, 711-720 (2018)

9. L. Zhou, G. Han, L. Liu, IEEE Access 5, 14380-14390 (2017)

10. O.S. Faragallah, H.M. Ibrahem, AEU Int. J. Electron. Commun. 70, 1034-1040 (2016)

11. A. Ramadhan, F. Mahmood, A. Elci, Int. J. Multimed. Appl. 9, 31-40 (2017)

12. G. Vivone, P. Braca, IEEE J. Ocean. Eng. 41, 1007-1019 (2016)

13. Y.F. Liu, J.D. Suo, X.M. Liu, B. Li, Appl. Mech. Mater. 701-702, 279-282 (2015)

14. H.Y. Cheng, H. Chien, Remote Sens. Environ. 188, 85-94 (2017)

15. C.P. Schwegmann, W. Kleynhans, B.P. Salmon, IEEE J STARS 8, 3329-3337 (2015)

16. F. Domma, F. Condino, B.V. Popović, J. Appl. Stat. 44, 2978-2993 (2016)

17. Y. Wang, D.D. Qu, C. Zhao, D. Yang, Photonic Sens. 8, 1-10 (2018)

18. A. Capria, E. Giusti, C. Moscardini, M. Conti, D. Petri, M. Martorella, F. Berizzi, IEEE Aerosp. Electron. Syst. Mag., 30-38 (2017)

19. F. Darwis, Y.N. Wijayanto, A. Setiawan, D. Mahmudin, A.N. Rahman, P. Daud, J. Phys. Conf. Ser. 1011, 012050 (2018) 